\title{
O DESENHO UNIVERSAL NA EDUCAÇÃO: NOVOS OLHARES DIANTE DA INCLUSÃO DO SER DEFICIENTE
}

\author{
EL DISEÑO UNIVERSAL EN LA EDUCACIÓN: NUEVAS MIRADAS ANTE LA \\ INCLUSIÓN DEL SER DEFICIENTE
}

\author{
THE UNIVERSAL DRAWING IN EDUCATION: NEW LOOK AT THE \\ INCLUSION OF THE DISABLED
}

\author{
Débora Cristina RICARDO ${ }^{1}$ \\ Lívia Fabiana $\mathrm{SAÇO}^{2}$ \\ Eliana Lúcia FERREIRA ${ }^{3}$
}

RESUMO: Nas últimas décadas, emerge de forma acelerada o contexto da reorganização e ressignificação da inclusão. Existe um movimento para o alcance das metas de uma educação acessível, a fim de promover a eliminação de barreiras buscando atender às necessidades de todos os estudantes que a frequentam, o que demanda a criação de circunstâncias convenientes, com a finalidade de que os discentes sintam-se acolhidos e envolvam-se, de forma efetiva, nas atividades educacionais. Assim, a acessibilidade institui transformações importantes na concepção do papel e das funções da educação superior, buscando uma forma de desenvolver práticas pedagógicas eficientes que assegurem a aprendizagem de todos. Numa perspectiva de que adotar o novo nem sempre significa repelir o velho, identificamos um modelo de educação dentro de uma concepção curricular heterogênea, reconhecendo os sujeitosalunos como protagonistas de seus destinos, como agentes de mudança, motivados por interesses e intencionalidades. Para tanto, é imprescindível repensar o uso da tecnologia assistiva (o velho modelo) dentro de um contexto maior, o do desenho universal (o novo modelo), promovendo uma melhor educação para os estudantes, nesse contexto, com diferentes mecanismos de ação.

PALAVRAS-CHAVE: Educação. Desenho universal. Inclusão.

RESUMEN: En las últimas décadas, emerge de forma acelerada el contexto de la reorganización y resignificación de la inclusión. Hay un movimiento para alcanzar las metas de una educación accesible, a fin de promover la eliminación de barreras buscando atender las necesidades de todos los estudiantes que la frecuentan, lo que demanda la creación de circunstancias convenientes, con la finalidad de que los discentes se se sienten acogidos y se involucran de forma efectiva en las actividades

\footnotetext{
${ }^{1}$ Universidade Federal de Juiz de Fora (UFJF), Juiz de Fora - MG - Brasil. Mestre em educação. E-mail: deboraricardo@hotmail.com.

${ }^{2}$ Universidade Federal de Juiz de Fora (UFJF), Juiz de Fora - MG - Brasil. Mestre em educação. E-mail: livia.saco@ufjf.edu.br

${ }^{3}$ Universidade Federal de Juiz de Fora (UFJF), Juiz de Fora - MG - Brasil. Faculdade de Educação Física e Desportos. Juiz de Fora. Docente na UFJF e Pesquisadora do projeto em rede "Acessibilidade no Ensino Superior" (Obeduc/Capes). E-mail:
} 
educativas. Así, la accesibilidad instituye transformaciones importantes en la concepción del papel y de las funciones de la educación superior, buscando una forma de desarrollar prácticas pedagógicas eficientes que aseguren el aprendizaje de todos. En una perspectiva de que adoptar lo nuevo no siempre significa repelir al viejo, identificamos un modelo de educación dentro de una concepción curricular heterogénea reconociendo a los sujetos-alumnos como protagonistas de sus destinos, como agentes de cambio, motivados por intereses e intencionalidades.Para ello, es imprescindible repensar el uso de la tecnología asistiva (el viejo modelo) dentro de un contexto mayor, el del diseño universal (el nuevo modelo), promoviendo una mejor educación para los estudiantes, en ese contexto, con diferentes mecanismos de acción.

PALABRAS CLAVE: Educación. Diseño universal. Inclusión.

ABSTRACT: In the last decades, the context of the reorganization and resignification of inclusion emerges in an accelerated way there is a movement to reach the goals of an accessible education, in order to promote the elimination of barriers in order to meet the needs of all the students who attend it, which demands the creation of convenient circumstances, so that the students feel welcomed and involved, in an effective way, in educational activities. Thus, accessibility institutes important transformations in the design of the role and functions of higher education, seeking a way to develop efficient pedagogical practices that ensure the learning of all. In a perspective that adopting the new does not always mean repelling the old, we identify a model of education within a heterogeneous curricular conception recognizing the student subjects as protagonists of their destiny, as agents of change, motivated by interests and intentionalities. Therefore, it is imperative to rethink the use of assistive technology (the old model) within a larger context, that of universal design (the new model), promoting a better education for students, in this context, with different mechanisms of action.

KEYWORDS: Education. Universal design. Inclusion.

\section{Introdução}

O termo Universal Design (UD) ou Design Universal (DU) surgiu na década de 1970, após a aprovação da Lei de Reabilitação Profissional (Vocational Rehabilitation Act), nos Estados Unidos (EUA), em 1973, proibindo qualquer forma de discriminação devido a questões de deficiência.

A primeira definição de Desenho Universal para a Aprendizagem surgiu no ano de 1984 em Boston, Massachusetts, nos Estados Unidos, no Center for Applied Special Technology (CAST), uma organização sem fins lucrativos formada por pesquisadores de educação para explorar novas tecnologias e proporcionar melhores experiências educacionais aos alunos com deficiência. 
Buscando adaptações para a realização da Educação Superior brasileira, trazemos nessa proposta o uso dos princípios da Disabilities, Opportunities, Internetworking, and Technology (DO-IT), uma unidade de serviços de tecnologia acessível da Universidade de Washington, D.C., EUA, que tem como objetivo promover o Desenho Universal para a aprendizagem, os ambientes e as instruções no âmbito da educação, mais especificamente da Educação Superior, tendo como principal autor Sheryl Brugstahler (DO-IT, 2016).

A Tecnologia Assistiva e o Desenho Universal são complementares no que tange a maximizar aspectos positivos na vida do deficiente (ROSE E MEYER (2002), EDYBURN (2010), CAMPO E MELLO (2015) e BURGSTAHLER (2015)). A legislação brasileira traz em seu Decreto 5.296/2004 uma abordagem interdisciplinar do estudo e da pesquisa relacionados à Tecnologia Assistiva, ao Desenho Universal e à Acessibilidade. Nesse Decreto, o DU é considerado como uma

[...] concepção de espaços, artefatos e produtos que visam atender, simultaneamente, a todas as pessoas, com diferentes características antropométricas e sensoriais, de forma autônoma, segura e confortável, constituindo-se nos elementos ou soluções que compõem a acessibilidade. (BRASIL, 2004).

A proposta do emprego do conceito de DU torna possível a transformação de uma realidade de segregação, de tutela, de paternalismo, para uma realidade de cidadania, de equiparação de oportunidades e de sociedade inclusiva (GALVÃO FILHO; GARCIA, 2012). A premissa principal desses conceitos é a de que os ambientes ou produtos sejam criados para serem usados por todas as pessoas, na sua máxima extensão possível, não necessitando de adaptações para atender às especificidades (GOVERNO DO ESTADO DE SÃO PAULO, 2010).

Com isso, o DU propõe soluções que compõem o escopo da acessibilidade, a fim de que haja a participação efetiva e a utilização dos recursos e/ou produtos por todos, independentemente da idade, do tamanho, da condição sensorial ou física, bem como qualquer outra característica que os indivíduos possam ter (CENTRO DE ESTUDOS E PESQUISA MUNICIPAL - CEPAM, 2008, p. 52 e 53). Segundo Campos e Mello (2015), devemos pensar em alternativas, tais como as diferentes formas de acesso ao conteúdo pedagógico; as diferentes formas de participação; os diferentes estilos de aprendizagem, habilidades e deficiências; além dos variados contextos de aprendizagem, quando transpostos para o contexto da Educação: um conjunto de 
princípios para o desenvolvimento de ambientes e de recursos pedagógicos que possibilitem processos de ensino e de aprendizagem ao maior número de pessoas.

Refletir acerca da educação para todos na Educação Superior vai ao encontro da premissa da Educação Inclusiva, que está fundamentada na concepção de direitos humanos, visando a garantir a todos a igualdade de oportunidades de aprendizagem e não somente às pessoas com deficiência (MIRANDA, 2002; BURGSTAHLER, 2015).

Enquanto o DU é mais voltado para as áreas de arquitetura e de construção, visando a produtos para uso "universal", o Universal Design for Learning (UDL), derivado do DU, é mais voltado para a área da Educação, visando à inclusão dos alunos por meio de um ambiente e de um currículo com possibilidades mais flexíveis. O UDL é uma área de estudo bastante recente no Brasil e, por isso, ainda há poucas publicações disponíveis. É preciso compreender que remover as barreiras físicas e tornar produtos já existentes em produtos verdadeiramente acessíveis pode-se tornar algo complexo e dispendioso. Isso é válido para os recursos educacionais, sendo assim, pensar na acessibilidade desde a concepção dos projetos é uma solução mais atrativa e necessária à sociedade contemporânea.

Essa procura de soluções individualizadas e inovadoras é alcançada ao se aplicar a concepção de flexibilidade inserida no currículo e, também, dando suporte aos docentes na melhoria do acesso ao conhecimento e à aprendizagem dentro da sala de aula (ROSE; MEYER, 2006; BURGSTAHLER, 2015). As ferramentas de suporte aos docentes fornecidas pelo DUA simplificam a avaliação do desempenho dos estudantes a respeito dos conhecimentos prévios, das potencialidades e das habilidades a adquirir. Além disso, essas ferramentas servem para detectar os facilitadores, as barreiras e os fatores ambientais que aumentam a participação do estudante no currículo geral (HITCHCOCK et al., 2002; BURGSTAHLER, 2015).

Aqueles que fazem uso do UDL consideram que o importante é ajudar o estudante a "aprender a aprender", a fim de que o aprendizado implícito ocorra de fato e que a avaliação não funcione como barreira, mas, ao contrário, que sirva para auxiliar no acesso, na participação e no desenvolvimento progressivo da aprendizagem. Um currículo acessível e inclusivo — do tipo que inclui suporte e várias formas de representação, de resposta e de expressão para um grupo maior de estudantes simplifica o acesso e o sucesso educativo, aumentando, assim, a qualidade do ensino.

Esses novos paradigmas que emergem na sociedade contemporânea são mais permeáveis à diversidade e estimulam a busca de novos caminhos de inclusão social. Os 
avanços na tecnologia são considerados por muitos especialistas como ações promissoras para novas concepções de inclusão e de acessibilidade dos indivíduos.

O caminho geral, rumo à uma política educacional inclusiva, difundiu as bases para solucionar, integralmente, as demandas educacionais da pessoa com deficiência. Incorporar essas vertentes jurídicas e sociais é relevante às novas tecnologias designadas a simplificar o acesso à educação desses estudantes. O Desenho Universal para Aprendizagem está na dianteira dessa nova era de concepções, tendências e possibilidades na educação especial.

Dentro desse aspecto, o DUA se configura como uma soma de princípios aplicáveis ao currículo, propiciando e estimulando o desenvolvimento de todos os estudantes em igualdade de oportunidades. O DUA significa, dessa maneira, uma mudança na forma de pensar a prática educacional em algumas formas básicas, com a flexibilização da maneira como a informação é apresentada, na maneira como os estudantes respondem ou expressam conhecimentos e habilidades e como os discentes estão engajados. Com isso, chega-se a uma diminuição das barreiras no ensino, propiciando acomodações condignas, o apoio aos desafios e, por fim, mantendo as expectativas de grandes realizações para todos os estudantes.

Portanto, os estudantes, tendo ou não deficiência, podem demonstrar distintas habilidades mentais, psicológicas e físicas, que ainda sofrem modificações no decorrer da vida, conforme o contexto e o passar dos anos. Nesse sentido, um modelo de DUA aplicado na criação de objetivos, métodos, materiais e avaliações para o processo de ensino-aprendizagem necessita ser flexível e habilitado a se adaptar às necessidades específicas de cada discente. Como resultado disso, o conhecimento é reforçado para todos os estudantes (FLETCHER, 2002; BURGSTAHLER, 2015).

$\mathrm{Na}$ perspectiva do DUA, as deficiências são entendidas como peculiaridades, como individualidades que necessitam ser consideradas nos projetos educacionais, com o intuito de que eles se tornem inclusivos, no sentido amplo, ou seja, que sejam para todos.

Além disso, em ambientes de aprendizagem, tais como os Institutos de Educação Superior (IES), o paradigma vigente de inclusão preconiza a ideologia de que nenhum indivíduo pode ser selecionado ou segregado em detrimento de suas diferenças, sejam elas orgânicas, sociais ou culturais. Isso nos leva à ruptura da ideia de um "estudante padrão" e à suposição de que haja um processo único de ensino-aprendizagem para todos os sujeitos (GLAT; NOGUEIRA, 2002). 
O paradigma da inclusão não defende a limitação a um modelo educacional único, mas se baseia em um outro modelo, em que o respeito e a aceitação da diferença, como requisitos característicos de uma sociedade plural, são considerados nas ações curriculares (GLAF; FERNANDES, 2005).

A utilização dos princípios do DUA propicia aos educadores a obtenção do referido objetivo, possibilitando uma delimitação para a compreensão de como desenvolver um currículo que, desde o princípio do projeto, atenda à demanda de todos os estudantes (CAST, 2011; UDL, 2014). Uma elaboração de condições de aprendizagens flexíveis, de simples utilização e cativantes para todos os estudantes, tornando o currículo mais acessível para os estudantes com incapacidades e, ao mesmo tempo, criando possibilidades de aprendizagem a todos.

Os princípios do DUA fundamentam-se na concepção de que o cérebro aprende por intermédio de três tipos de redes neuronais, o que tem sido decisivo para os estudos e os avanços das Neurociências (ROSE; MEYER, 2002).

O cérebro de cada indivíduo é único, e através da aprendizagem e modos singulares denota estilos, ritmos de aprendizagem e modos de padronização individuais. Rose e Meyer (2002) abordam, ainda, que somos diferentes devido ao nosso cérebro, que é singular no Reconhecimento (aprender "o que"), na Estratégia (aprender “como") e no Afeto (aprender “o porquê").

Entender como a aprendizagem se realiza a nível cerebral possibilita-nos a fomentar ambientes de aprendizagem relevantes, concretos, vivos e provocadores, que reúnam a enorme capacidade dos seus cérebros para aprender, o que está fundamentalmente relacionado com os princípios e diretrizes orientadoras do DUA.

Através das redes de reconhecimento ocorre o reconhecimento da informação a ser compreendida, específica para apreender e atribuir sentido aos padrões que nós vemos. Elas nos auxiliam a reconhecer e a entender as informações, as ideias e os conceitos (VYGOTSKT,1962; ROSE; MEYER, 2002). Especializadas na forma como sentimos e damos significado aos padrões que vemos. Elas nos possibilitam identificar, entender e processar as concepções, princípios e informações adquiridas pelos canais sensoriais (CAST, UDLCENTER, 2016).

As redes de estratégias processam informações e são especializadas em criar e supervisionar padrões mentais e motores. Elas também nos possibilitam planejar, executar e monitorar ações e habilidades (VYGOTSKT,1962; ROSE; MEYER, 2002).

Complementando os princípios, a rede afetiva engloba o engajamento na tarefa 
de aprender, estimulam ao interesse no aprendizado e no mundo que nos rodeia especialistas em avaliar padrões e conceder a eles um sentido emocional (VYGOTSKT,1962; ROSE; MEYER, 2002).

Mesmo levando em consideração que todos os cérebros possuem as mesmas características gerais, individualmente eles são fundamentalmente diferentes. Compreender as funções específicas das redes de reconhecimento, estratégica e afetiva pode nos auxiliar a entender as limitações e as capacidades de cada estudante.

Os três princípios do DUA atendem à heterogeneidade, aos diferentes estilos de aprendizagem, fazendo com que as funções e as competências cognitivas de qualquer estudante, com ou sem deficiência, possam ser melhoradas, aperfeiçoadas, por intermédio de uma nova visão do currículo e da viabilidade de educar cada discente. Esses princípios podem ser aplicáveis em todos os níveis, uma vez que se verifica uma discordância substancial entre a população estudantil atual e os currículos padronizados (ROSE; MEYER, 2002).

\section{Repensando a prática inclusiva na Educação do Ensino Superior: caminhos para a acessibilidade}

Tendo em vista que o acesso a um sistema educacional inclusivo leva em conta a disponibilização de recursos de apoio que garantam a acessibilidade de todos os estudantes, temos no Brasil como reflexo os recursos do Programa Incluir e do Decreto n. 6.571/2008 (BRASIL, 2008), que exigiu a estruturação de núcleos de acessibilidade em todas as IES federais brasileiras. A implantação desses Núcleos de Acessibilidade veio para atender e cumprir uma exigência legal e, também, para que todos os alunos possam se desenvolver e aprender de forma autônoma. É de responsabilidade das Universidades proporcionar não só os recursos, como também o conjunto de ações e programas que possibilitem a aprendizagem e a acessibilidade dos estudantes. Pavão e Bortolazzo (2016) afirmam que:

Não se pode em tempo algum garantir que as pessoas aprendam, posto ser a aprendizagem o resultado das ações dos sujeitos no mundo. $\mathrm{O}$ que se pode é organizar ambientes, implantar programas e desenvolver políticas que atendam as demandas de inclusão, aprendizagem e acessibilidade desses sujeitos em situação educacional. A implementação de núcleos de acessibilidade nas instituições de nível superior, em sendo uma das ações que, de modo mais próximo, visam ao atendimento dessas complexas questões que envolvem a aprendizagem na educação Superior. 
É notório o crescente número de deficientes no meio acadêmico a partir do século XXI, emanados pelo processo de democratização da educação superior associado às políticas públicas brasileiras; visa-se nesse processo a inclusão da pessoa com deficiência a partir da promoção de acessibilidade, como demonstra os dados do gráfico 1, exemplificando nesse contexto, a partir de dados da Universidade Federal de Juiz de Fora (UFJF, 2009).

\section{Gráfico 1: Evolução de alunos com deficiência matriculados nos cursos de graduação da UFJF no período de 2001 a 2016}

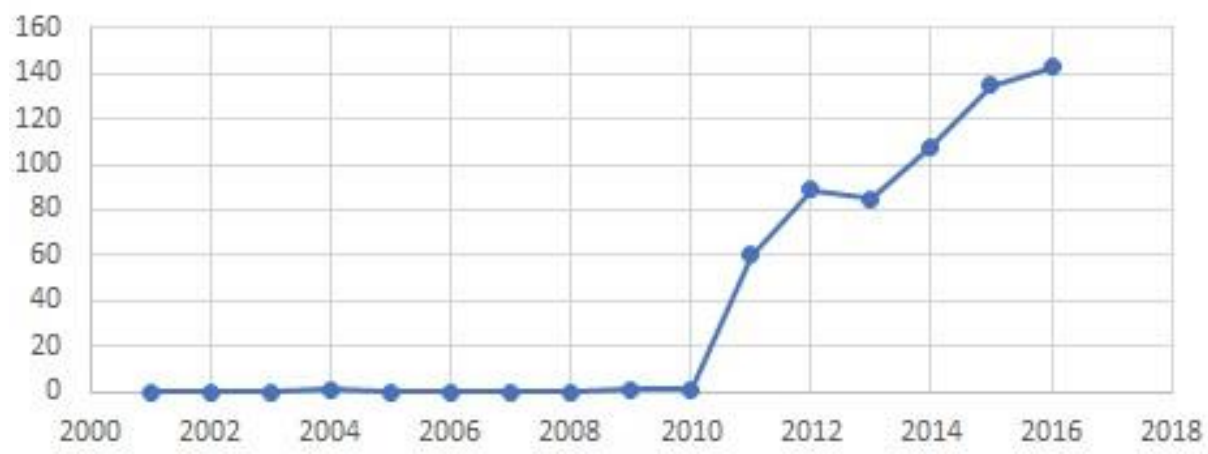

Fonte: Elaborado pelos autores

Segundo Almeida, Bellosi e Ferreira (2015), as questões pautadas na ausência quase total de pessoas com deficiência até o ano de 2010 perpassa por mudanças na coleta de dados realizada pelo Instituto Nacional de Estudos e Pesquisas Educacionais Anísio Teixeira - INEP (2013) após 2009, reflexo dos recursos do programa Incluir e do decreto $\mathrm{n}^{\circ}$. 6571/2008 somados às políticas públicas implementados no Brasil nos últimos anos (BRASIL, 2008).

Repensar propostas visando não somente ao acesso, mas à permanência dos mesmos, com equidade, igualdade e qualidade, no ensino superior, é de suma importância e de extrema responsabilidade social.

A concepção de acessibilidade como estado de alcance e ruptura de barreiras físicas e sociais serve para a equiparação de oportunidades e para a qualidade de vida de todos, efetivando, principalmente, a inclusão das pessoas com deficiência nos diversos meios sociais, como o trabalho, a escola e o lazer.

Esses componentes, dentro de um contexto mais específico que, em nosso caso, 
é a educação, devem garantir um ensino diferenciado, que combine diferentes estratégias de aprendizagem, ao mesmo tempo em que seja capaz de diversificar e de transformar apresentação e representação de conteúdos, utilizando-se de diferentes metodologias e de múltiplos recursos, a fim de que o estudante consiga demonstrar o que aprendeu, através de diversos meios de ação. O DUA, dessa forma, aumenta o impacto da educação inclusiva, pois amplia um conceito de instituição educacional que conhece e torna viável as diferenças entre os estudantes, propiciando, assim, instruções específicas que estimulem os discentes a aprender e a utilizar requisitos característicos do Século XXI, de maneira a articular, com eficácia, inclusive as tecnologias atuais.

Essa procura de soluções individualizadas e inovadoras é alcançada ao se aplicar a concepção de flexibilidade inserida no currículo escolar e, também, dando suporte aos docentes na melhoria do acesso ao conhecimento e à aprendizagem dentro da sala de aula (ROSE; MEYER, 2006). Nessa proposta, o DUA fornece ferramentas de suporte aos docentes que simplificam a avaliação do desempenho dos estudantes a respeito dos conhecimentos prévios, das potencialidades e das habilidades a adquirir. Além disso, essas ferramentas servem para detectar os facilitadores, as barreiras e os fatores ambientais que aumentam a participação do estudante no currículo geral (HITCHCOCK et al., 2002).

Elas consideram que o importante é ajudar o estudante a "aprender a aprender", a fim de que o aprendizado implícito ocorra de fato e que a avaliação não funcione como barreira, mas, ao contrário, que sirva para auxiliar no acesso, na participação e no desenvolvimento progressivo da aprendizagem. Um currículo acessível e inclusivo, do tipo que inclui suporte e várias formas de representação, de resposta e de expressão para um grupo maior de estudantes, simplifica o acesso e o sucesso educativo, aumentando, assim, a qualidade do ensino.

Hoje, observamos que o desenvolvimento tecnológico nos beneficia constantemente, o que nos coloca diariamente à disposição de novas ferramentas que favorecem e facilitam o nosso cotidiano. Esse mesmo desenvolvimento tecnológico fornece ferramentas ou práticas que ampliam, agilizam e promovem respostas aos problemas funcionais encontrados pela pessoa com deficiência (Bersch, 2009).

Nesse contexto, as Tecnologias Assistivas (TA) são soluções tecnológicas especialmente projetadas para ajudar as pessoas com deficiência na superação das barreiras em seu ambiente, aumentando a sua oportunidade de independência. TA são únicas e pessoais, sendo cuidadosamente projetadas, equipadas e adaptadas aos pontos 
fortes e fracos de cada pessoa (Rose et al., 2005).

A Tecnologia Assistiva é empregada ao nível de cada discente, a fim de ajudá-lo a superar as barreiras do currículo e dos ambientes de aprendizagem. Com o Desenho Universal essa proposta se amplia no contexto das propostas curriculares e ambientes, minimizando ao máximo as barreiras tradicionais de aprendizagem.

Não há um método único de aprendizagem para tornar cada estudante um especialista no reconhecimento, porém, um meio que seja adequado a diversas estratégias de ensino pode auxiliar o sucesso nessa área. Um acertado suporte para propiciar o reconhecimento é oferecer inúmeros exemplos, utilizando textos, imagens (visualização) ou situação real (manipulação). O uso do computador e de outros recursos também pode ser um apoio para os docentes possibilitarem alternativas de apresentação da informação nos diversos formatos. Valorizar e utilizar o processamento ativo dos conhecimentos prévios dos estudantes, além de aumentar a transferência e a generalização das aprendizagens, também são escolhas eficazes para potencializar o reconhecimento, desenvolvendo estudantes competentes e experientes, o que está de acordo com o primeiro princípio do DUA (ROSE; MEYER; HITCHCOCK, 2005).

Essas redes neurais são especializadas em gerar e supervisionar os padrões mentais e motores. Elas nos possibilitam planejar, realizar e controlar ações e habilidades. Ao responder alguma coisa, usamos as redes estratégicas, uma vez que elas correspondem ao "como" da aprendizagem. As redes em questão estão localizadas no lobo frontal, que engloba grande parte do córtex, e efetuam funções de extrema complexidade, como o processamento sensorial, o motor e o da cognição. Além disso, ainda possibilitam que todas as ações e os pensamentos sejam percebidos conscientemente. A parte da frente do lobo frontal, o córtex pré-frontal, está, de modo direto, relacionado à estratégia de estabelecer quais sequências de movimento acionar e como ordenar e avaliar seu resultado. As suas utilidades parecem abarcar o pensamento abstrato e criativo, a fluidez do pensamento e da linguagem, a vontade, a firmeza para ação, o juízo social e a atenção seletiva.

Os procedimentos de ensino associados a redes de estratégia fundamentam-se na antecipação das barreiras à aprendizagem estratégica, bem como na escolha de materiais e de práticas que sejam flexíveis e permitam superar essas barreiras. Essas são alternativas, como os recursos digitais, as simulações de computador e a realidade virtual, e possibilitam, também, o feedback à aprendizagem do estudante, o que é fundamental para formar indivíduos estratégicos e bem direcionados. Importa, ainda, 
criar níveis graduais de base ao desempenho/participação do estudante e, assim, simplificar a monitoria do processo de ensino-aprendizagem por parte do discente, diferenciando métodos de resposta e de percurso (ROSE; MEYER; HITCHCOCK, 2005).

As redes afetivas estão relacionadas ao interesse e à motivação, possibilitando avaliar os padrões, conceder-lhes significado emocional e envolvê-los em atividades/aprendizagem com o mundo que nos cerca. Os instrumentos que controlam os níveis de atividade nas diversas partes do encéfalo e as bases de impulsos da motivação, especialmente a direcionada para o processo de aprendizagem, assim como as sensações de prazer ou punição, são executadas, em grande parte, pelas regiões basais do cérebro, as quais, reunidas, integram o Sistema Límbico. As redes afetivas são "o porquê" da aprendizagem, das tarefas e das ideologias que nos desafiam. Elas estão circunscritas ao Sistema Límbico e relacionadas, essencialmente, à regulação dos processos emocionais. Trata-se de um sistema no formato de anel cortical, ininterrupto, que circunda as formações inter-hemisféricas (ROSE; MEYER, 2006).

Quando os estudantes não estão interessados na aprendizagem, os esforços para auxiliá-los serão irrelevantes. É por esse motivo que o terceiro princípio é o mais valorizado de todos. De fato, a afetividade assume a maior relevância no processo de ensino-aprendizagem, uma vez que a forma como os estudantes são motivados para aprender representa um elemento definitivo, estando o DUA na vanguarda dessa concepção. (ROSE; MEYER, 2006). Para poder ganhar estudantes motivados e determinados, os docentes têm que ter conhecimento de como educar para a autonomia, acatar as escolhas individuais dos discentes, intensificar o sentido de colaboração em diferentes ambientes de aprendizagem, proporcionar a participação social e o espírito de responsabilidade na comunidade, oferecer níveis ajustáveis/autênticos de estímulo e de prêmios/recompensas, valorizar a habilidade individual de ultrapassar dificuldades, por intermédio da autoavaliação e da reflexão. Os estudantes repetiram, ainda, a importância da escolha de conteúdos e de recursos digitais (ROSE; MEYER, 2002).

O conjunto de saberes resultantes da Neurociência são, nomeadamente, os sistemas envolvidos na aprendizagem. De acordo com vários autores (CAST, 2016; COUREY et al., 2012; ROSE; MEYER, 2002; BURGSTAHLER, 2015), a Neurociência oferece uma base sólida para o entendimento de como o cérebro aprende e de como é possível proporcionar um ensino eficiente. Esse conjunto de saberes demonstram que a aprendizagem é um processo multifacetado. 


\section{Considerações finais}

O caminho geral rumo à política educacional mais inclusiva difundiu as bases para solucionar, integralmente, as demandas educacionais da pessoa com deficiência. Incorporar essas vertentes jurídicas e sociais é relevante às novas tecnologias designadas a simplificar o acesso à educação desses estudantes. Apesar de o Desenho Universal estar na dianteira dessa nova era de concepções, de tendências e de possibilidades na educação especial, constata-se a ausência da inclusão desse modelo nas Universidades e Instituições Federais, apesar de constar nas políticas públicas de acessibilidade e de inclusão do MEC.

No aspecto educacional, o DU se configura como uma soma de princípios aplicáveis ao currículo, propiciando e estimulando o desenvolvimento de todos os estudantes em igualdade de oportunidades. O DU significa, dessa maneira, uma mudança na forma de pensar a prática educacional em algumas formas básicas, com a flexibilização da maneira como a informação é apresentada, na maneira como os estudantes respondem ou expressam conhecimentos e habilidades e como os discentes estão engajados. Com isso, deve-se chegar a uma diminuição das barreiras no ensino, propiciando acomodações condignas, o apoio aos desafios e, por fim, mantendo as expectativas de grandes realizações para todos os estudantes.

Na perspectiva do DU, as deficiências são entendidas como peculiaridades, como individualidades que necessitam ser consideradas nos projetos educacionais, com o intuito de que eles se tornem inclusivos no sentido amplo, isto é, para todos.

Além disso, o paradigma vigente de inclusão das Universidades Federais Brasileiras preconiza a ideia de que nenhum indivíduo pode ser selecionado ou segregado em detrimento de suas diferenças, sejam elas orgânicas, sociais ou culturais, exclui o modelo educacional único e limitado, baseando-se em um modelo que considera o respeito e a aceitação da diferença como requisitos característicos de uma sociedade plural.

Vale ressaltar que uma universidade plural para todos tem o compromisso de garantir oportunidades iguais para cada um, satisfazendo às suas diferentes capacidades, salientando, ao máximo, o seu potencial individual e a sua inclusão no grupo. As ajudas técnicas, compreendidas como instrumentos de efetivação dessa igualdade de oportunidades, necessitam ser desenhadas, de modo a garantir a sua colocação na realidade complexa e, por tantas vezes, contraditória, do ambiente formal de educação. 
Dessa forma, criar, adaptar ou aplicar qualquer solução de Tecnologia Assistiva deve demandar e responsabilizar a teia de relações que o discente estabelece na IES, seja com os docentes, seja com os profissionais técnicos de apoio, seja com a família ou com os amigos, que colaboram, dessa forma, para explorar o seu potencial como indivíduo, sem criar segregações ocultas.

\section{REFERÊNCIAS}

ALMEIDA, J. G. A.; BELOSSI, T. C.; FERREIRA, E. L. Evolução da matrícula de pessoas com deficiência na educação superior brasileira: subsídios normativos e ações institucionais para acesso e permanência. Revista Ibero-Americana de Estudos em Educação. v.10, n. esp.1, p. 643-660, 2015. Disponível em:

<seer.fclar.unesp.br/iberoamericana/article/download/7917/5433>. Acesso em: 01 jul. 2017.

BRASIL. Decreto n⿳ 6.571/2008. Dispõe sobre o Atendimento Educacional Especializado. 17 set. 2008. Disponível em: <http://www.planalto.gov.br/ccivil_03/_ato2007 2010/2008/Decreto/D6571.htm>. Acesso em: 16 mar. 2017.

BERSCH, R. Introdução à tecnologia assistiva. 2005, p. 3. Disponível em: <http://www.cedion-line.com.br/artigo_ta.html>. Acesso em 12 dez. 2016.

BURGSTAHLER, Sheryl E. Universal design in higher education: from principles to practice. $2^{a}$ edição, Harvard, 384 p., 2015.

CAMPOS, T.; MELLO, M. A. O desenho universal e a tecnologia assistiva como potencializadores dos processos de ensino e aprendizagem: Parte II, 2015.

Disponível em: <http://technocare.net.br/portal/wpcontent/uploads/2015/05/desenho_universal.pdf>. Acesso em: 10 mar. 2017.

CAST. Design for learning guidelines. APA Citation: CAST, 2011. Disponível em: <http://www.udlcenter.org/sites/udlcenter.org/files/guidelines.pdf >. Acesso em: $17 \mathrm{abr}$. 2017.

CAST. About universal design for learning, 2016. Disponível em:

<http://www.cast.org/our-work/about-udl.html\#.WUQ86OvyuM9>. Acesso em: 16 jun. 2017.

CENTRO DE ESTUDOS E PESQUISA MUNICIPAL. Fundação Prefeito Faria Lima. Acessibilidade nos municípios: como aplicar o decreto 5296/04, 2008. Disponível em: <www.cepam.sp.gov.br/arquivos/acessibilidade>. p. 56-58. Acesso em: 12 mar. 2017.

COUREY, J. S.; TAPPE, P.; SIKE, J.; LEPAGE, P. (2012). Improved lesson planning with universal design for leaning (UDL). Teacher education and special education, $v$. 36, n. 1, p. 7-27. Disponível em:

<http://citeseerx.ist.psu.edu/viewdoc/download?doi=10.1.1.822.5148\&rep=rep1\&type= pdf>. Acesso em: 25 fev. 2017. 
EDYBURN, D.L. Would you recognize universal design for learning if you saw it? Ten propositions for new directions for the second decade of UDL. Learning disabilities quarterly, p. 33-41, 2010.

FLETCHER, V. Design universal: design para o século 21. [s.1.]: Griffith University, 2002.

GALVÃO FILHO, T. A.; DAMASCENO, L. L. As novas tecnologias e a tecnologia assistiva: utilizando os recursos de acessibilidade na educação especial. In: III Congresso Ibero-Americano de Informática na Educação Especial. Anais. Fortaleza, MEC, 2012.

GLAT, R.; FERNANDES, E. M. Da educação segregada à educação inclusiva: uma breve reflexão sobre os paradigmas educacionais no contexto da educação especial brasileira. Revista inclusão: MEC/SEESP, v. 1, n. 1, p. 35-39, 2005. Disponível em: $<$ http://forumeja.org.br/sites/forumeja.org.br/files/Da\%20Educa\%C3\%A7\%C3\%A3o\% 20Segregada\%20\%C3\%A0\%20Educa\%C3\%A7\%C3\%A3o\%20Inclusiva.pdf >. Acesso em: 20 jun. 2017.

GLAT, R.; NOGUEIRA, M. L. de L. Políticas educacionais e a formação de professores para a educação inclusiva no Brasil. Revista Integração. v. 24, ano 14; Brasília: MEC/SEESP, p. 22-27, 2002.

GOVERNO DE SÃO PAULO. Desenho universal: habitação de interesse social no Estado de São Paulo. 2010, p.14-35. Disponível em: <http://www.mpsp.mp.br/manualdesenho-universal>. Acesso em: 16 mar. 2017.

HITCHCOCK, C.; et al. Providing new access to the general curriculum. Universal Design for Learning. In: Teaching exceptional children, p. 8-35, 2002.

MEYER, A.; ROSE, D. H.; GORDON, D. Universal design for learning: Theory and practice. Wakefield: CAST Professional Publishing, 2014.

INEP (Instituto Nacional de Estudos e Pesquisas Educacionais “Anísio Teixeira”).

Censo da educação superior: 2011 - resumo técnico. Brasília: Inep, 2013.

MIRANDA, A.S. Recomendações de acessibilidade digital em cursos de educação a distância via web para portadores de deficiência visual. Dissertação (Mestrado) Universidade Federal de Santa Catarina, Florianópolis. 2002.

PAVÃO, S. M. de O.; BORTOLAZZO, J. Ações de atenção à aprendizagem no ensino superior. Santa Maria: Laboratório de Pesq. e DOC. CE,1. ed. UFSM, 2015.

Disponível em:

$<$ http://coral.ufsm.br/anima/images/Aes\%20de\%20Ateno\%20\%20Aprendizagem\%20no $\% 20$ Ensino\%20Superior.pdf>. Acesso em: 05 maio 2017.

ROSE, D.H.; Meyer, A.; Hitchcock, C. The universally designed classroom:

Accessible Curriculum and Digital Technologies. Cambridge: Harvard Education, 2002. 
ROSE, D.H.; Meyer, A.; Hitchcock, C. A practical reader in universal design for learning. Cambridge, MA: Harvard Education Press, 2006.

UNIVERSAL DESIGN FOR LEARNING - UDL. Guidelines - version 2.0. 2014. Disponível em: <www.udlcenter.org>. Acesso em: 15 fev. 2017.

VYGOTSKY, L.S. Thought and Language. Cambridge, MA: MIT Press.1962.

Disponível em: <http://www.instructionaldesign.org/theories/social-development.html>. Acesso em 15 fev. 2016.

UNIVERSIDADE FEDERAL DE JUIZ DE FORA. Coordenação de acessibilidade educacional, física e informacional. Caefi. Juiz de Fora, 2009. Disponível em: <http://www.ufjf. br/acessibilidade/caefi/>. Acesso em: 10 maio 2017.

\section{Como referenciar este artigo}

RICARDO, Débora Cristina.; SAÇO, Lívia Fabiana.; FERREIRA, Eliana Lúcia. O desenho universal na educação: novos olhares diante da inclusão do ser deficiente. Revista Ibero-Americana de Estudos em Educação, Araraquara, v. 12, n. esp. 2, p. 1524-1538, ago./2017. Disponível em: <http://dx.doi.org/10.21723/riaee.v12.n.esp.2.10083>. E-ISSN: 1982-5587.

Submetido em: 08/07/2017

Aprovação final em: 20/08/2017 